\title{
Video Article \\ A Volumetric Method for Quantification of Cerebral Vasospasm in a Murine Model of Subarachnoid Hemorrhage
} \author{
C. Thal ${ }^{4}$ \\ ${ }^{1}$ Department of Neurosurgery, Medical Center of the Johannes Gutenberg - University \\ ${ }^{2}$ Department of Neuroradiology, Medical Center of the Johannes Gutenberg - University \\ ${ }^{3}$ Platform for Biomaterial Research, Medical Center of the Johannes Gutenberg - University \\ ${ }^{4}$ Department of Anesthesiology, Medical Center of the Johannes Gutenberg - University \\ *These authors contributed equally
}

Axel Neulen ${ }^{*}$, Michael Kosterhon ${ }^{* 1}$, Tobias Pantel ${ }^{* 1}$, Stefanie Kirschner ${ }^{2}$, Hermann Goetz $^{3}$, Marc A. Brockmann ${ }^{2}$, Sven R. Kantelhardt $^{1}$, Serge

Correspondence to: Axel Neulen at axel.neulen@unimedizin-mainz.de, Serge C. Thal at thal@uni-mainz.de

URL: https://www.jove.com/video/57997

DOI: doi:10.3791/57997

Keywords: Neuroscience, Issue 137, Subarachnoid hemorrhage, SAH, mouse, cerebral vasospasm, micro computed tomography, endovascular casting

\section{Date Published: $7 / 28 / 2018$}

Citation: Neulen, A., Kosterhon, M., Pantel, T., Kirschner, S., Goetz, H., Brockmann, M.A., Kantelhardt, S.R., Thal, S.C. A Volumetric Method for Quantification of Cerebral Vasospasm in a Murine Model of Subarachnoid Hemorrhage. J. Vis. Exp. (137), e57997, doi:10.3791/57997 (2018).

\section{Abstract}

Subarachnoid hemorrhage $(\mathrm{SAH})$ is a subtype of hemorrhagic stroke. Cerebral vasospasm that occurs in the aftermath of the bleeding is an important factor determining patient outcome and is therefore frequently taken as a study endpoint. However, in small animal studies on $\mathrm{SAH}$, quantification of cerebral vasospasm is a major challenge. Here, an ex vivo method is presented that allows quantification of volumes of entire vessel segments, which can be used as an objective measure to quantify cerebral vasospasm. In a first step, endovascular casting of the cerebral vasculature is performed using a radiopaque casting agent. Then, cross-sectional imaging data are acquired by micro computed tomography. The final step involves 3-dimensional reconstruction of the virtual vascular tree, followed by an algorithm to calculate center lines and volumes of the selected vessel segments. The method resulted in a highly accurate virtual reconstruction of the cerebrovascular tree shown by a diameter-based comparison of anatomical samples with their virtual reconstructions. Compared with vessel diameters alone, the vessel volumes highlight the differences between vasospastic and non-vasospastic vessels shown in a series of SAH and sham-operated mice.

\section{Video Link}

The video component of this article can be found at https://www.jove.com/video/57997/

\section{Introduction}

Aneurysmatic subarachnoid hemorrhage (SAH), a subtype of hemorrhagic stroke, is a common disease in neurointensive care units. Besides early brain injury (EBI), which comprises cerebral damage caused by the bleeding event itself, another important factor determining patient outcome is delayed cerebral ischemia $(\mathrm{DCl})$, defined by clinical deterioration through impaired cerebral perfusion or cerebral infarction not associated with interventional or surgical procedures ${ }^{1,2,3}$. Important mechanisms contributing to DCl are vasospasms of large cerebral vessels on the one hand; on the other hand, microcirculatory dysfunction with vasospasm of microvessels and microthrombosis, and ischemia related to cortical spreading depressions play a role (reviewed in Madonald 2014 ${ }^{1}$ ). Therefore, diagnosis of vasospasm of the large cerebral vessels is crucial in clinical practice and displays an important endpoint in many clinical and experimental studies.

Despite the fact that the features of vasospasm in murine SAH models are not directly transferable to the human patient, murine models of SAH related vasospasm have been of growing significance in the last years. In these models SAH is induced by endovascular filament perforation ${ }^{4,5,6,7,8}$, transection of cisternal vessels ${ }^{9}$, or injection of blood into the CSF ${ }^{10,11,12}$. In contrast to large animal models of SAH which were traditionally designed to study vasospasm ${ }^{13}$, murine models have the great advantage that numerous transgenic mice strains are available. This makes them an excellent tool for studying molecular mechanisms leading to vasospasm and DCl. However, determination of cerebral vasospasm in mice is challenging. This is because in contrast to large animal models in which vasospasm can be examined using clinical imaging techniques, in vivo imaging to analyze cerebral vasospasm in mice is not yet available. Therefore, vasospasm is commonly determined using either histological sections ${ }^{10,11}$ or microscopically after casting of cerebral vessels ${ }^{7,9,12}$. However, these techniques have the disadvantage that vessel diameters are examined at defined points only.

Based on a previous study ${ }^{7}$, this manuscript presents a method for objective and reproducible analysis of vasospasm in a murine SAH model. The method is based on perfusion and casting of the cerebral vessels, ex vivo micro-CT scanning, digital reconstruction of the vessel tree, and subsequent evaluation of volumes of entire cerebral vessels. 


\section{Protocol}

The animal experiments were approved by the responsible animal care committee (Landesuntersuchungsamt Rheinland-Pfalz) and carried out in accordance with the German Animal Welfare Act (TierSchG). All applicable international, national, and institutional guidelines for the care and use of animals were followed.

In this study, male C57BL6 mice (age 10-12 weeks) were used. In brief, subarachnoid hemorrhage was induced by endovascular filament perforation under anesthesia with isoflurane. The left external carotid artery was prepared surgically. Then, a filament was inserted into the external carotid artery and advanced intracranially through the internal carotid artery which was perforated at the carotid T, inducing a subarachnoid hemorrhage. A rise in intracranial pressure was taken as an indicator of successful endovascular perforation. A detailed protocol of an endovascular filament perforation model of SAH in mice has been published by others ${ }^{8,14}$.

\section{Perfusion and Endovascular Casting}

1. In this study, perfusion was performed 72 hours after induction of SAH. Induce anesthesia by intraperitoneally injecting $5 \mu \mathrm{g} / \mathrm{g}$ body weight (bw) midazolam, $30 \mathrm{ng} / \mathrm{g} \mathrm{bw}$ fentanyl, and $0.5 \mu \mathrm{g} / \mathrm{g}$ bw medetomidin. Continue only after a sufficient anesthesia level has been reached, which is confirmed by the absence of reactions to pain stimuli.

2. Open the thorax, puncture the left ventricle with a $21 \mathrm{G}$ cannula, open the right atrium, and clamp the descending aorta as described elsewhere ${ }^{15}$.

3. Perform a transcardiac perfusion using the following solutions: (i) Dulbecco's Phosphate Buffered Saline containing $\mathrm{MgCl}_{2}$ and $\mathrm{CaCl}_{2}$ at $\mathrm{pH}$ 7.4 with $1 \mathrm{~g} / \mathrm{L}$ glucose, and (ii) $4 \%$ paraformaldehyde solution.

1. Start the perfusion with solution (i) for 2 minutes and continue with solution (ii) for 4 minutes.

2. Infuse the solutions at a temperature of $37^{\circ} \mathrm{C}$ and using a pressure-controlled pump with variable perfusion rate to perfuse with a constant pressure of $70 \mathrm{mmHg}$, which was found to be the optimal perfusion pressure to analyze vasospasm in mice ${ }^{16}$. Avoid a loss of pressure when switching from solution (i) to solution (ii).

4. After perfusion with solutions (i) and (ii), continue perfusion for 20 minutes at room temperature with radiopaque casting agent (see Table of Materials) at a constant rate of $0.2 \mathrm{ml} / \mathrm{min}$.

5. Allow for curing of the radiopaque casting material at $4{ }^{\circ} \mathrm{C}$ overnight. Then remove the brain from the skull as previously described ${ }^{17}$, transfer the sample to $4 \%$ PFA solution, and store the sample at $4{ }^{\circ} \mathrm{C}$ until micro $\mathrm{CT}$ scanning.

\section{Micro Computed Tomography}

1. Place the brain in the center of a plastic tube with a blunt anatomical forceps. Choose a tube with a slightly larger diameter than the sample to ensure that the object does not move during image acquisition. Use gauze to close the tube.

2. Attach the plastic tube to the micro-stepping motor on the computer-navigated-control (CNC) positioning system in the $\mathrm{X}$-ray cabin, in which the object is rotated around its horizontal axis.

3. Align the sample in the field-of-view under X-ray radiography. To achieve the maximal magnification, place the object as close as possible to the $\mathrm{x}$-ray source and maximize the distance to the detector as far as possible.

4. Use a step-and-shoot image acquisition protocol with the following scan parameters: set exposure time to $1 \mathrm{~s}$ for each projection to optimize the signal-to-noise ratio (SNR), tube voltage $80 \mathrm{kV}$ (current $38 \mu \mathrm{A}), 360^{\circ}$ rotation resulting in 1,000 projections.

5. For reconstruction of RAW-Data use a filtered back projection algorithm applying the Shepp-Logan filter with a matrix of $1024 \times 1024 \times 1024$ voxels using reconstruction software (see Table of Materials). For further analysis import the resulting DICOM data into 3D visualization software (see Table of Materials).

\section{3-dimensional Reconstruction of the Intracranial Vascular Tree and Determination of Vessel Volumes}

Note: Background information on the functions of the visualization software can be found using the function help.

1. Import Dicom data into visualization software using the function Import.

2. Visualize the vessel tree with the function Volren. Choose the visualization threshold so that the large cerebral arteries are depicted in sharp outlines. It is important to use the same visualization threshold for all data sets belonging to the experimental series.

3. Virtually dissect the basal cerebral arteries (Circle of Willis) with the function VolumeEdit by surrounding the vessels with the cursor. Then virtually dissect the vessel segment to be analyzed. Therefore, rotate the 3-dimensional model of the vascular tree in order to precisely separate all small branches from the main artery. It is essential for the further analysis to delete all vessels except the vessel segment to be analyzed.

4. Apply the function Autoskeleton with threshold set to visualization threshold, which generates a center-line-based SpatialGraph.

5. Then, apply the function SpatialGraphToLineSet to create a line set. Divide the line set into its single subsegments by manually choosing the single subsegments with the cursor and clicking on "split". This step is crucial in order to calculate the volumes of the single subsegments.

6. Use the function LineSetToSpatialGraph to create a Spatial Graph again.

7. Use the function SpatialGraphStatistics to determine length, volume, and diameter of each subsegment. For color-coded visualization representing the course of the vessel diameter, use the function SpatialGraphView. Set segment coloring to "thickness", which correlates with the vessel diameter. It is important to choose the same color map for all datasets belonging to the experimental series. 
8. Add the lengths of the subsegments to determine which subsegments are to be included in the further analysis. In the present study we evaluated a vessel segment consisting of $1 \mathrm{~mm}$ of the internal carotid artery proximal of the carotid T and $2.5 \mathrm{~mm}$ of the middle cerebral artery distal of the carotid T. Then add the volumes to determine the vessel volume of the defined vessel segment.

\section{Representative Results}

\section{Virtual reconstruction of the 3-dimensional intracranial vascular tree}

The 3-dimensionally reconstructed intracranial vascular trees provided a highly accurate vascular anatomy (Figure 1). To assess the accuracy, we performed a diameter-based comparison between vessel diameters determined microscopically and from the 3-dimensional virtual reconstructions at 2 anatomically defined points (1: left middle cerebral artery (MCA) $1 \mathrm{~mm}$ distal of the carotid T; 2 : right MCA $1 \mathrm{~mm}$ distal of the carotid T). For microscopic determination of vessel diameters, a high-resolution camera (Infinity X-21, Deltapix) with DeltaPix Insight software version 2.0.1 calibrated to a micrometer scale was used. For this evaluation, 10 brain samples ( $5 \mathrm{SAH}, 5$ sham) were analyzed. These were from a series of 12 mice, in 7 of which an SAH was induced, while 5 underwent sham surgery ( 2 animals of the SAH group died on postoperative days 1 and 2, respectively). There were no significant differences between the diameters determined microscopically and virtually, indicating an accurate virtual reconstruction of the intracranial vascular anatomy (microscopic determination vs. virtual reconstruction, mean \pm standard error: left MCA $150 \pm 9 \mu \mathrm{m} v s$. left MCA $150 \pm 8 \mu \mathrm{m}$; right MCA $153 \pm 8 \mu \mathrm{m} v s .154 \pm 9 \mu \mathrm{m}$, see Figure 2).

\section{Quantification of cerebral vasospasm in mice with SAH}

To quantify cerebral vasospasm, (i) the volume of a predefined representative $3.5 \mathrm{~mm}$ vessel segment, consisting of $1 \mathrm{~mm}$ internal carotid artery (ICA) and $2.5 \mathrm{~mm}$ MCA on the left, and (ii) the vessel diameters at 2 anatomically defined points (left and right MCA) were determined in brain samples from the SAH and sham-operated animals $(n=5)$. The vessel volume was significantly lower in SAH compared to sham (36 $\pm 4 \mathrm{~nL} v s$. $71 \pm 9 \mathrm{~nL}, p<0.05$ ). Vessel diameters were lower in SAH compared to sham (left MCA: $140 \pm 11 \mu \mathrm{m} v \mathrm{~s}$. $160 \pm 10 \mu \mathrm{m}, p=0.11$; right MCA: 130 $\pm 16 \mu \mathrm{m}$ vs. $158 \pm 13 \mu \mathrm{m}, p<0.05$; see Figure 3), while the level of significance was only reached for analysis of the right MCA.
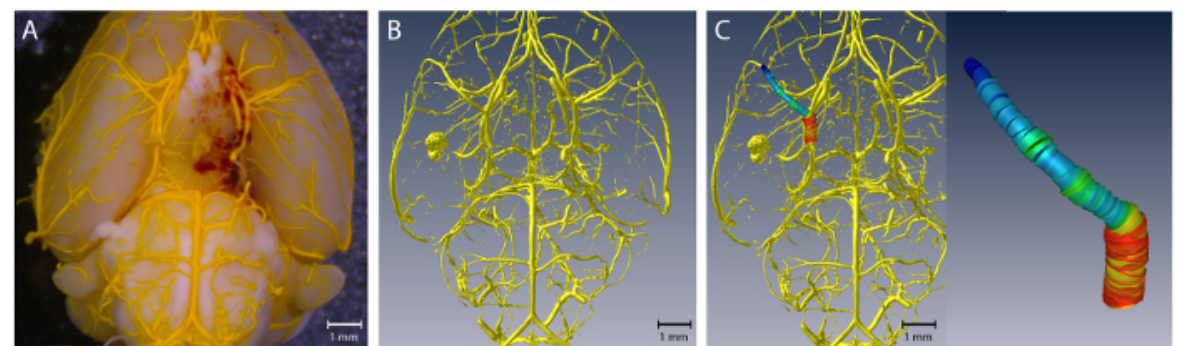

Figure 1. Virtual reconstruction of the intracranial vascular tree. (A) shows a representative brain sample; (B) shows the corresponding virtually reconstructed vascular tree. (C) Color-coded visualization of the diameter of the left MCA. Please click here to view a larger version of this figure.

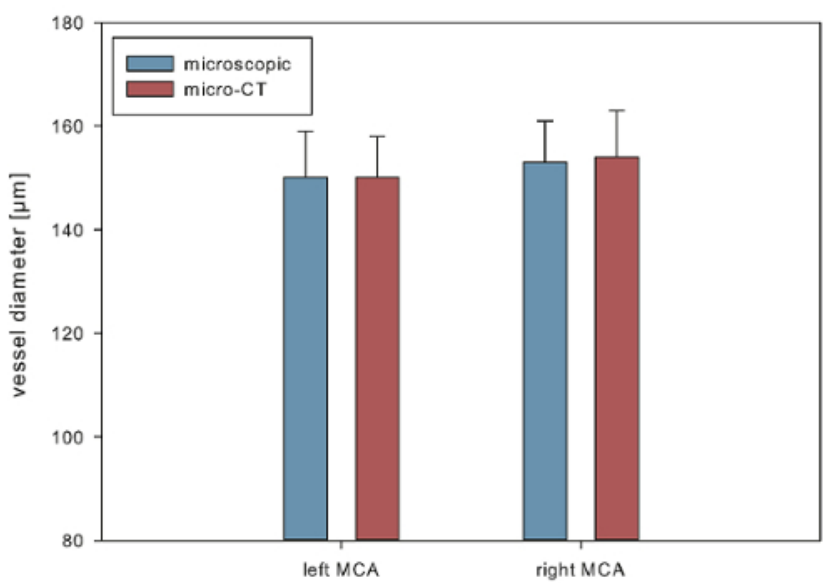

Figure 2. Accuracy of the digital reconstruction of the vasculature. Mean diameters measured from 3D reconstructed brain samples compared with those determined microscopically. Data are shown as mean \pm standard error of the mean. Please click here to view a larger version of this figure. 
A Vessel diameter in SAH and sham animals

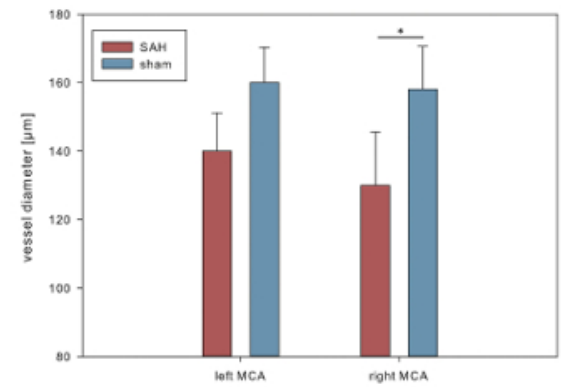

B Vessel yolume in SAH and sham animals

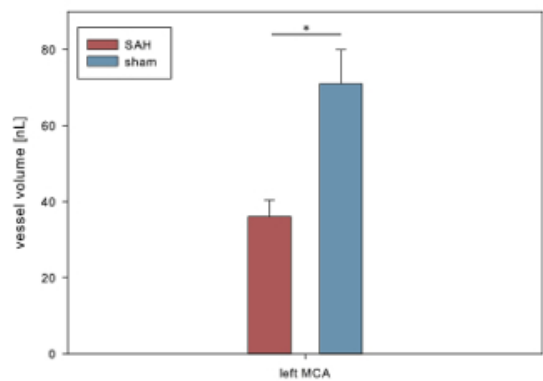

Figure 3. Vessel volume and vessel diameter after SAH. (A) Comparison of MCA diameters measured from 3D reconstructed vasculature in $\mathrm{SAH}$ and sham mice. (B) Vessel volume in SAH and sham mice. Data are shown as mean \pm standard error of the mean. ${ }^{*} p<0.05$. Please click here to view a larger version of this figure.

\section{Discussion}

Murine SAH models are an important tool for basic SAH research. Cerebral vasospasm is frequently used as an endpoint in experimental studies investigating the mechanisms leading to $\mathrm{DCl}$ after $\mathrm{SAH}^{9,11}$. However, quantification of cerebral vasospasm in mice or other small animal models of SAH is challenging. Commonly, vasospasm is quantified by ex vivo determination of vessel diameters at defined anatomical points after endovascular perfusion and casting ${ }^{7,9,12}$ or by determination of the circumference of defined vessels on histological sections ${ }^{10,11}$. However, these methods have some disadvantages: Vasospasm is evaluated only at defined anatomical points; vasospasm of neighboring vessel segments may escape evaluation. Histological artifacts present another source of errors. Furthermore, the evaluation can be rather subjective, because the exact position where the vessel diameter is measured is determined by the investigator.

The goal was therefore to establish a method that quantifies cerebral vasospasm by calculating the vessel volume of entire cerebral vessel segments from cross-sectional imaging data ${ }^{7}$. The most important advantage of the volumetric method presented here is that entire vessel segments can be examined. This avoids the need of definition of a point where the vessel diameter is measured. A further advantage of the evaluation of whole vessel segments is that it presumably presents a more objective parameter to quantify vasospasm than determination of vessel diameters at defined points where vasospasm of the more proximal or distal vessel may escape evaluation. Digital representation of vessel diameters using a color code allows an intuitive estimation of the degree of vasospasm. Furthermore, volumetric evaluation leads to larger differences between vasospastic vessels compared to evaluation of vessel diameters as shown in the representative results. The virtual reconstruction achieved with the method presented here reflects the vascular anatomy accurately. This is shown by the evaluation of the representative series, in which vessel diameters measured microscopically and from the digital reconstructions were similar, reproducing the observations of a previous study ${ }^{7}$. However, despite its advantages, further studies are needed to evaluate whether or not the method presented here is superior to conventional methods of vasospasm analysis.

A limitation of the method presented here is that it affords more time compared to microscopic analysis of casted brain samples or histological analysis (micro CT scanning time 90 minutes per brain sample, data processing $45 \mathrm{~min}$ per brain sample). Furthermore, the availability of micro CT scanners may limit its application. The number of animals examined here was sufficient to demonstrate the feasibility of the protocol described in this manuscript. However, if the protocol should be used in treatment studies, animal numbers would have to be calculated based on the expected effects on vessel volumes and diameters. Another limitation of this and other studies using murine SAH models is that vasospasm is determined ex vivo. This makes longitudinal studies impossible that investigate baseline values before SAH induction and vasospasm at different time points. Although studies have demonstrated that it is possible to depict the anatomy of the large intracranial vessels of mice in vivo using magnetic resonance tomography ${ }^{18}$, computed tomography angiography ${ }^{19}$, or digital subtraction angiography ${ }^{20}$, these methods, to our knowledge, have not yet been used to analyze cerebral vasospasm in murine SAH models in vivo. Of note, the digital reconstruction of the cerebral vasculature with subsequent volumetric evaluation of cerebral vasospasm presented here is not limited to the use on ex vivo micro CT data. If high resolution vascular cross sectional brain imaging in mice should become available in the future, it could be used to perform a volumetric analysis of vasospasm in vivo.

\section{Disclosures}

The authors have nothing to disclose.

\section{Acknowledgements}

Parts of this study are part of the doctoral thesis of T. Pantel, presented to the Medical Faculty of the Johannes Gutenberg-University of Mainz. The study was supported by the Friedhelm Frees Stiftung and by the Stiftung Neurochirurgische Forschung (grants to A.N.).

\section{References}

1. Macdonald, R. L. Delayed neurological deterioration after subarachnoid haemorrhage. Nature Reviews Neurology. 10 (1), $44-58$ (2014).

2. Dorsch, N. A clinical review of cerebral vasospasm and delayed ischaemia following aneurysm rupture. Acta Neurochirurgica Supplement. 110 (Pt 1), 5-6 (2011). 
3. Vergouwen, M. D. et al. Definition of delayed cerebral ischemia after aneurysmal subarachnoid hemorrhage as an outcome event in clinical trials and observational studies: Proposal of a multidisciplinary research group. Stroke. 41 (10), 2391-2395 (2010).

4. Friedrich, B. et al. $\mathrm{CO} 2$ has no therapeutic effect on early microvasospasm after experimental subarachnoid hemorrhage. Journal of Cerebral Blood Flow \& Metabolism. 34 (8), e1-6 (2014).

5. Friedrich, B., Muller, F., Feiler, S., Scholler, K., \& Plesnila, N. Experimental subarachnoid hemorrhage causes early and long-lasting microarterial constriction and microthrombosis: An in vivo microscopy study. Journal of Cerebral Blood Flow \& Metabolism. 32 (3), 447-455 (2012).

6. Terpolilli, N. A. et al. Nitric oxide inhalation reduces brain damage, prevents mortality, and improves neurological outcome after subarachnoid hemorrhage by resolving early pial microvasospasms. Journal of Cerebral Blood Flow \& Metabolism. 36 (12), 2096-2107 (2016).

7. Neulen, A. et al. A segmentation-based volumetric approach to localize and quantify cerebral vasospasm based on tomographic imaging data. PLoS One. 12 (2), e0172010 (2017).

8. Schuller, K., Buhler, D., \& Plesnila, N. A murine model of subarachnoid hemorrhage. Journal of Visualized Experiments. (81), e50845 (2013).

9. Altay, T. et al. A novel method for subarachnoid hemorrhage to induce vasospasm in mice. Journal of Neuroscience Methods. 183 (2), 136-140 (2009).

10. Momin, E. N. et al. Controlled delivery of nitric oxide inhibits leukocyte migration and prevents vasospasm in haptoglobin $2-2$ mice after subarachnoid hemorrhage. Neurosurgery. 65 (5), 937-945; discussion 945 (2009).

11. Froehler, M. T. et al. Vasospasm after subarachnoid hemorrhage in haptoglobin 2-2 mice can be prevented with a glutathione peroxidase mimetic. Journal of Clinical Neurocience. 17 (9), 1169-1172 (2010).

12. Lin, C. L. et al. A murine model of subarachnoid hemorrhage-induced cerebral vasospasm. Journal of Neuroscience Methods. 123 (1), $89-97$ (2003).

13. Marbacher, S., Fandino, J., \& Kitchen, N. D. Standard intracranial in vivo animal models of delayed cerebral vasospasm. British Journal of Neurosurgery. 24 (4), 415-434 (2010).

14. Feiler, S., Friedrich, B., Scholler, K., Thal, S. C., \& Plesnila, N. Standardized induction of subarachnoid hemorrhage in mice by intracranial pressure monitoring. Journal of Neuroscience Methods. 190 (2), 164-170 (2010).

15. Ghanavati, S., Yu, L. X., Lerch, J. P., \& Sled, J. G. A perfusion procedure for imaging of the mouse cerebral vasculature by X-ray micro-CT. Journal of Neuroscience Methods. 221 70-77 (2014).

16. Parra, A. et al. Mouse model of subarachnoid hemorrhage associated cerebral vasospasm: methodological analysis. Neurological research 24 (5), 510-516 (2002).

17. Boulay, A. C., Saubamea, B., Decleves, X., \& Cohen-Salmon, M. Purification of Mouse Brain Vessels. Journal of Visualized Experiments. (105), e53208 (2015).

18. Marjamaa, J. et al. Mice with a deletion in the first intron of the Col1a1 gene develop dissection and rupture of aorta in the absence of aneurysms: High-resolution magnetic resonance imaging, at 4.7 T, of the aorta and cerebral arteries. Magnetic Resonance in Medicine. 55 (3), 592-597 (2006).

19. Schambach, S. J. et al. Ultrafast high-resolution in vivo volume-CTA of mice cerebral vessels. Stroke. 40 (4), $1444-1450$ (2009).

20. Figueiredo, G. et al. Comparison of digital subtraction angiography, micro-computed tomography angiography and magnetic resonance angiography in the assessment of the cerebrovascular system in live mice. Clinical Neuroradiology. 22 (1), 21-28 (2012). 